\title{
Features in the Emergence and Reemergence of Infectious Diseases, Gain-of-Function Research and Geopolitics
}

\author{
Chrysanthus Chukwuma Sr* \\ The Chrysanthus Centre for Future-Oriented Studies, Centre for Future-Oriented Studies, CTR Future-Oriented Ltd, Nigeria \\ *Corresponding author: Chrysanthus Chukwuma Sr, The Chrysanthus Centre for Future-Oriented Studies, Centre for \\ Future, Oriented Studies, CTR Future-Oriented Ltd, Abakaliki, Ebonyi State, Nigeria
}

\section{ARTICLE INFO}

Received: 幽 December 12, 2021

Published: 㓞 January 04, 2022

Citation: Chrysanthus Chukwuma Sr. Features in the Emergence and Reemergence of Infectious Diseases, Gain-ofFunction Research and Geopolitics. Biomed J Sci \& Tech Res 40(5)-2022. BJSTR. MS.ID.006500.

Keywords: Vaccines; Treatment; AIDS; HIV; SARS-CoV-2; Vulnerable Populations

\begin{abstract}
Research and publication are relevant from perspectives in the management of local and global health because disease is perspicuously a geopolitical issue ostensibly linked to gain-of-function research where health diplomacy undergirds present and future global functionalities regarding the emergence and reemergence of infectious diseases. These have generated vehement reactions with propensity for extreme geopolitics and gain-of-function natural and anthropogenic activities. Geopolitical parameters and gain-of-function issues impact on the social determinants of health and vice versa. The convening and convergence of countries for unprecented epidemic or pandemic treaty settings or other formulations to confront emerging and reemerging infectious diseases will afford considerable opportunities concerning challenges in action, preparedness and response. Provisions are pertinent for legal instruments, effective and efficient systems to curb future threats and outbreaks of infectious diseases.
\end{abstract}

Abbreviations: GOF: Gain-Of-Function; GOFROC: Gain-of-Function Research of Concern; LMICs: Low- and Middle-Income Countries; GoF: Gain-of-Function Research

\section{Introduction}

The application or utilization of gain-of-function, GoF or gainof-function research, GoFR in association or absence of geopolitics or geopolitical activities has created concerns in the emergence and reemergence of infectious diseases [1]. TheSARS-CoV-2 or COVID-19 $[1,2]$ Omicron ( $\mathrm{Nu}$ or $\mathrm{Xi}$ ) is a nascent or newfangled Variant of Concern. This designation as may be applicable to other nascent or newfangled microorganisms, such as subunit variant BA. 2 of the Omicron COVID-19 genre denotes sharing of genome sequences, informing the WHO of initial cases and clusters, conducting field, laboratory investigations and assessment to explicate or elucidate efficiency, effectivity, epidemiology, public health, medical and nonpharmaceutical inputs, severity, transmissibility, clinic pathological correlates and overall impacts of these microorganisms as they pertain to the global population, especially vulnerable populations. It is imperative to resist "travel apartheid" in a pandemic that is not an endemic situation as in the current ban on select countries due to the COVID-19 Omicron variant. In the guise of preventing COVID-19, the world must tread with caution on Emergency Use Authorization and booster doses of vaccines, especially with the advent of the subunit variant BA.2 Omicron COVID-19. Although, gain-of-function research enables testing of new scientific theories, development of novel technologies, discoveries and targets for treatment of infectious diseases, it is a territory to be treated with caution.

\section{Global Conspectus on the Impacts of Infectious Diseases}

In recent decades, there have been exacerbating outbreaks of infectious diseases with grave emergence and reemergence worldwide. These incorporate the severe acute respiratory syndrome coronaviruses [1,2], swine flu, Middle East respiratory syndrome coronavirus, the Zika virus and the Ebola virus disease disseminating across international boundaries; thus, ushering in an era of unprecedented climate, demographic and technologic 
spatiotemporal variations [1] in both rural and urban precincts with escalating threats to hunger/food security, environment, health and socioeconomic activities [1,3,4]. These pose grave threats which require countries with vast resources and conscionable geopolitical imperatives to contribute and enhance global health and environmental security to stem the increased risk in the emergence and reemergence of novel infectious diseases [5-7]. All nations continue facing the threats of an avalanche of emerging and reemerging infectious disease epidemics and pandemics.

These have resulted in widespread escalating global concerns due to their excruciating and debilitating effects $[1,8]$ with the inextricable linkage of infectious diseases and geopolitical stability without an iota of consideration for international boundaries or borders. Understanding geopolitical determinants and gain-offunction research will enhance evidence and imperatives for the improvement of preventing and treating emerging and reemerging infectious diseases globally. Protagonists of international health, policy and security have perspicuously enhanced health perspectives and interventions in association with political stability and gain-of-function studies to address the disproportionate global perturbations and impact of the SARS-CoV-2 Omicron variant and other microbial variants as they reflect on strategies to balance public and medical modalities in the pandemic devoid of stigmatisation regarding the origin(s)/source(s) as in HIV/AIDS controversies [9].

\section{Gain-of-Function Research and Geopolitical Attributes}

Gain-of-Function research is capable of exposing perspicuous mutation occurring in defined viruses, permits easier community surveillance, and identification of the genesis of the mutations, contemporaneous production of compatible and veritable vaccines $[6,7]$ and feasible treatments. Thus GoF research connotes the serial presentation of microorganisms whereby their host tropisms, immunogenicity and virulence are augmented via the inducement of specific pressure to a defined culture [10].

CRISPR, a gene editing technology is feasible coupled with selective serial passaging in the investigation of the functionality of defined genes on protein expression and roles of organisms as well as the biological mechanisms undergirding the transmission and replication of viruses [10]. Nascent SARS-CoV-2 variants having mutations in the spike glycoprotein emanated independently in numerous locales within ostensibly significant functionalities. These aggregated mutations in the 501Y, K417N and E484K were detected in the receptor-binding domain, RBD including mutations in the N-terminal domain, NTD [11]. The nascent SARS-CoV-2 or COVID-19 Omicron variant, B.1.1.529 (Nu, Xi) was detected through genomic sequencing.

\section{Vaccines and Treatment}

Exploration of vaccines and treatment modalities as well as trending strategic scenarios in the context and perspective of erstwhile epidemics and pandemics provides valuable applications for the prevention, control and treatment of emerging and reemerging infectious diseases. This means an upward review for inter alia compulsory application of face masks, practical sustained manual hygiene, social distancing, assembly in well-ventilated ambients and other pertinent non-pharmacological interventions, compliance to preventative strategies to restrict the dissemination of the newfangled variants [6,7] per gain-of-function research and geopolitical interventions. Statistical equivalence tend to indicate that vaccines have been protective against the presenting coronavirus variants. The mRNA vaccines by Moderna and PfizerBioNtech are more effective than the Astrazeneca variety. Animals are liable to contract SARS-CoV-2, and viral reservoirs can revert same to humans. However, the pharmaceutical industries are faced with paucity of data in the choice of viruses for immunization. A newfangled oral antiviral drug, Molnupiravir for the effective treatment of the COVID has been produced by the Merck and Ridgeback Biotherapeutics [12], and it is incumbent on developed countries with abundant resources to distribute these vaccines to poorer countries and vulnerable populations worldwide [6,7].

Due to emerging variants, the COVID-19 pandemic has placed the production of a veritable vaccine in an intricately complex situation as the pharmaceutical industries have limited data for the immunization against any particular coronavirus. It is considered that the gain-of-function research whereby there is certain genetic manipulation culminated in more deleterious delta variant of SARSCoV-2, the etiologic agent of COVID-19 [6, 7]. Although, this remains speculative, the gain-of-function standard on the conspiracy and collaboration of China and the NIH of USA will be protracted even as the source, origin, socioeconomics, geopolitics and legal implications of SARS-CoV-2 are sustained or controverted by Gainof-function research of concern, GOFROC as a discretionary term [13]. With regard to pharmaceutical drugs, Ibuprofen, also called Advil and Motrin is a common non-steroidal anti-inflammatory anti-pyretic therapeutic agent, additionally used for pain mitigation, fever, swelling and redness [14]. It was suggested that ibuprofen was an inappropriate treatment for certain symptoms in COVID-19 patients because SARS-CoV-2 was detected to bind to target cells through ACE2 in the respiratory apparatus [15]. Thus, it was suggested that ACE2-stimulating drugs, ACE inhibitors and ibuprofen aggravate the risk in the severity of morbidity and mortality of COVID-19 [16]; and, as such, not amenable in COVID-19 suspected cases [17]. However, it has not been proven that ibuprofen is associated with symptom derangement, prolongs 
illness, or that resultant or concomitant inflammations mask COVID-19 severity, interfere in certain immune response or elevate the viral infection [14].

These controversies that ibuprofen and other NSAIDs are not veritable choices in the treatment of symptoms in COVID-19 patients may be related to bias of retrospective short-term studies. Armchair scientists, researchers and medical practitioners are liable to misinterpret, misrepresent, omit or mislead authentic bioinformatics and spatiotemporal variations [1,14] geopolitical activities and gain-of-function in SARS-CoV-2 or COVID-19 emerging and reemerging variants. The unpredictable and unprecedented accelerated dissemination of these nascent problems present novel challenges and opportunities for the production, formulation, administration and regulation of vaccines and other therapeutic pharmacological and non-pharmacological approaches $[6,7,14,18]$.

\section{Natural and Anthropogenic Factors in Infectious Disease Progression}

Socioeconomic, natural, anthropogenic and other related constructed systems, such as geopolitics and gain-in-function research modalities have contributed to deleterious effects of certain microorganisms including the HIV/AIDS and COVID-19 or SARS-CoV-2 pandemic. These have impacted on the environment, social welfare, wellbeing and quality of life, biodiversity, ecological systems, governmental policy, governance [19], ecosystem cadastre [3], primary healthcare structure $[20,21]$ with concomitant social hysteria [22] in this COVID-19 pandemic era. These are presenting expansive effects of epidemics and pandemics across domestic and international borders or boundaries of countries or regions which have altered the trajectories of emerging and reemerging infectious diseases. The eradication or mitigation of upsurge in variants of infectious diseases may discontinue ecological interference of diverse epidemics and pandemics, such as HIV/AIDS, coronavirus, Cryptosporidium, Cystoisospora, Acanthamoeba, Ebola virus, Lassa fever, and yellow fever infections and infestations [1]

Contributing factors to emerging and reemerging infectious diseases include expansive increase of human population, migration, rapid increases in internecine conflicts, hunger [23], geopolitical situations, deforestation, and abuse of pharmacological agents $[24,25]$ as well as the complexity of interactive events of anthropogenic and natural interplay in the emergence of novel microbial agents, and reemergence of erstwhile infectious diseases presumed to have been eradicated. Natural occurrences and anthropogenic activities have expansively contributed to facilitate the emergence, reemergence and dissemination of infectious diseases both locally and globally with their extant constraints and challenges as they fit into the environmental, public health, sociological, economic, salient and latent spatiotemporal and pecuniary burdens [22] associated with developmental and geopolitical activities.

\section{Exploring Converging Spheres of Emerging and Reemerging Infectious Diseases and Microorganisms}

Researchers tend to agree that within the past four decades, there have been remarkable global perspectives in taking cognizance and responding to threats of infectious diseases and harmful microbiota, especially since the SARS outbreak of 2003 [1,26,27]. The geopolitics of the presenting COVID-19 pandemic intersects in an inextricably complex global and transboundary trajectory that challenges global governance, policy and international cooperation. The COVID-19 pandemic has exposed the inherent dangers in the frailty and fragility of international cooperation of discrete and disparate national boundaries and territorial integrities or other collective responsibilities for sharing and distributing public health supplies, deciding on local and global strategic issues, excoriating and exacerbating pressures on food security [28], vaccines [6,7], scarce resources $[29,30]$, disruption of population density and climate change, especially in the least developed countries. These also pertain to the developed countries with the hardest hit of the COVID-19 pandemic exposed to environmental perturbations, such as poor atmospheric/air quality, public health and climate issues or challenges for coordinated response in a multidimensional strategy [31].

The immunodeficiency syndrome is a presentation of relatively inextricably-linked severe disorders due to the human immune deficiency virus, HIV1 [9]. AIDS is mainly a disorder in erstwhile healthy persons without perspicuous underlying iatrogenic etiology. Controversies have been enmeshed in the origin [32], incidence and prevalence of HIV, the social implications [9] and legal status have been extensively debated [33]. Devoid of effective and efficient vaccination, there will be incessant dissemination of the AIDS virus across international boundaries worldwide, there is no extant conclusive evidence that the AIDS virus emanated from sub-Saharan Africa, or that the progenitor virus was extant in Central Africa [9]. Even by 2030; the African region may not terminate the AIDS scourge as a public health threat because the continent continues to fall short of the pertinent $75 \%$ dimunition in nascent HIV infections and 81\% 2020 dimunition in AIDSassociated mortality [34]. These are evident, although, a vast majority of persons are aware of their HIV status and treatment schedules or rates.

Nascent HIV infections and AIDS-associated mortality are not concomitantly diminishing. The African continent, and especially, the sub-Saharan Africa sub-region cannot afford to be detracted in the urgent quest and focus to curb the inequities which conduct AIDS, other epidemics and pandemics. Despite the challenges and 
issues, it is evident that remarkable progress has been achieved or achievable against HIV in recent decades and in the future regarding the decrement of morbidity and mortality rates in the entire continent due to ardent and strigent measures as well as consumption of antiretroviral drugs. It is imperative to employ equitable access to treatment, uninterrupted services, global sharing and solidarity which constitute essential ingredients to harness and curb HIV/AIDS, COVID-19 or SARS-CoV-2 and other emerging and reemerging deleterious microbiome. The Cryptosporidium parasite with the ensuing infection of cryptosporidiosis continues unabated to constitute both environmental and public health problems in the category of emerging and reemerging diseases globally $[35,36]$.

The correlates of protective immunity and the parasitosis of the Cryptosporidium spp in both humans and animals have ostensibly confounded policy makers and researchers alike; and its prevention has involved configuring disparate or converging modes of research and policies which may have impacted, and continue to impact on geopolitics and gain-of-function issues not yet unravelled in the public domain. These tendencies are invariably manifest in cystoisosporiasis, erstwhile referred to as isosporiasis [37]. Globally, especially in tropical and sub-tropical ambients, the infection due to the etiologic agent, Cystoisospora belli, C. belli is common in immunocompromised persons, such as HIV-infected/AIDS patients. Cystoisosporiasis, HIV/AIDS and SARSCoV-2 patients exhibit clinicopathological correlates pertinent to elucidate associated comorbidities and mechanisms of these disorders. Geopolitics and gain-of-function tinkering are liable to obfuscate or mask the contracting and dissemination of these diseases resulting in gross neglect of the disparate pathological influences and manifestations among vulnerable populations [37].

Cystoisoporiasis and several other protozoal infestations and infections, such as microsporidiosis exclusively or in comorbidity with AIDS [38] are emerging and reemerging infectious diseases [1] prevalent in low- and middle-income countries, LMICs than in high income countries. Amongst other protozoal infections, cystoisosporiasis patients may not be more susceptible to SARSCoV-2/COVID-19 but the probable geopolitics and gain-offunction trajectories with resultant variants pose dimensions of concern. The epidemiology and clinicopathological features of $C$. belli are significant for routine surveillance of the parasite in immunosuppressed patients [37] for sustainable geopolitics and gain-of-function of concern as well as related anthropogenic interventions in gain-of-function research.

Thus, in the ocular apparatus, SARS-CoV-2, otherwise alluded to as the coronavirus 2019 or COVID-19 has precipitated health repercursions of perturbing unprecedented magnitude or dimensions $[39,40]$ with deterioration of the ophthalmic system. It has caused susceptible individuals suffering from Acanthamoebic keratitis to seek cogent healthcare due to increased risk of COVID-19 infections via wearing of contact lenses [41]. There are stringent efforts to prioritize community capacity in the surveillance and curbing of Acanthamoeba keratitis in the environ [42] to avoid severe ophthalmic diseases, such as anterior uveitis and conjuctivitis as well as the attendant consequences of geopolitical and gain-of-function issues.

\section{Socioeconomic Effects of Emerging and Reemerging Infectious Diseases}

The alleviation or eradication of local and global poverty as well as hunger [43] with adequate health provisions, sustainable development and environment is a daunting task in the face of epidemics and pandemics of emerging and reemerging infectious diseases as depicted, for instance, in the scourges of the swine flu, HIV/AIDS, Ebola virus, SARS-CoV-2 or COVID-19. This is more so as we take into cognizance the UN's 17 Goals in the face of constricted resources and bourgeoning human populations inhabiting Planet Earth. With current abject poverty and hunger amongst vulnerable populations in tropical and sub-tropical Africa, Asia including Latin America in contrast to ostensibly resource-endowed North America and Europe, there are greater extant socioeconomic and healthcare deprivations and diminished wealth acquisition and resource control in the COVID-19 pandemic [29,30].

The SARS-CoV-2 or COVID-19 variants have configured or undergone gain-of-function to expose local and universal geopolitics with resultant excruciating hunger [22]. Indubitably, the COVID-19 pandemic with other emerging and reemerging, and non-comparatively salient infectious diseases have exposed the non-pragmatic preparedness, conscience and justice in the imposed geopolitics of local and global institutions driven by inter alia the disruption, and dimunitive capacity of small- and medium-sized enterprises. It is pertinent to predict the impact and resurgence of epidemics and pandemics of infectious diseases and their variants of concern in order to rescue the human population and businesses from extreme dissipation.

\section{Discussion}

Gain-of-Function research (GoF research, GoFR) medically implies genetic alteration of an organism to augment the biological functionalities of gene products, such as pathogenesis, transmissibility or microbiome host range and targets to optimally predict the emergence and reemergence of infectious diseases with concomitant development or formulation of vaccines and therapeutic agents [44]. Inasmuch as diverse entities, such as policy makers and research scientists may differentially explicate Gain-of-Function research, it may contextually connote genetically as mutations which grant a gene, RNA or protein newfangled potentialities or expression in contradistinction to Loss-of-Function that depicts the resultant dimunitive capacity of a gene to access 
its deficiency, thereof [45]. As it presents expansive advancements in global health, gain-of-function research poses biosafety or biosecurity and governance concerns which definitely neccesitate legislative and regulatory measures regarding the emergence [46] and reemergence of infectious diseases in convergence with both domestic and international standards [47], undergirding environmental legislation and citizen participation [48] for sustainable development [49] to obviate risks, dire repercussions, intransigent, unconscionable and irresponsible research as may affect biosafety and biosecurity [47].

The epidemics and pandemics associated with emerging and reemerging infectious diseases with their superimposed geopolitics and gain-of-function issues portend far-reaching impacts on the human population both locally and globally [1]. Persons having acute and chronic health conditions are invariably at differential risks of morbidity and mortality from the sequelae of inter alia SARS-CoV-2/COVID-19 and HIV/AIDS [50]. The superimposed social distance to flatten the curve in nascent cases negatively depict the status of the economy, thus, intersecting extant health perturbations, such as HIV/PWH within vulnerable populations. The clinicopathological correlates in these diseases may contribute in unravelling or elucidating contemporaneous prevention, treatment, vaccines [51] and control of geopolitics and gain-offunction natural and anthropogenic occurrences in the emergence and reemergence of infectious diseases $[1,37,52]$.

\section{Conclusion}

The emergence and reemergence of infectious diseases have posed expansively grave threats globally. Invariably, numerous disparate and inextricably-linked factors, such as demographic spatiotemporal variations, disruption of ecosystem cadaster, inappropriate, incomprehensible and dysfunctional health systems as well as intersecting geopolitical exhibitions, natural and anthropogenic gain-of-function presentations. Presently, of all the emerging and reemerging infectious diseases, it is pellucid that SARS-CoV-2 as the causative agent of the COVID-19 pandemic has assumed center stage in global politics and investigations, especially geopolitics and gain-of-function research with its neverending variants of concern, such as the nascent and prevailing Omicron. Even though, the Omicron variant is a recent discovery, governments, economies and health systems have responded immensely to curtail an unpredictable and unprecedented adverse dissemination of events.

The rapid global dissemination of this nascent variant may be precarious due to spatiotemporal deficient genomic sequences with probable interlaced non detection of several cases, and masking of the presence and/or effects of concomitant emergence of other variants and infectious diseases. With the simultaneous ban and restriction on foreign travels, the world economy has begun to take a plunge for the worst. Numerous economies are vulnerable to the dissemination of the adverse emergence and reemergence of infectious diseases presenting a bleak outlook and test for economic policies and governance. The invariable podcasts and calculus depict incapacitated and ignorant countries in dire need on how to cope with emerging and reemerging infectious diseases by obviating the adverse effects of geopolitics and gain-of-function research.

\section{References}

1. Chukwuma Sr C (2018) Bioinformatics-base and determinants in the spatiotemporal variations of emerging and reemerging infectious diseases. Journal of Ancient Diseases \& Preventive Remedies 6(02): 10.

2. Vicidomini S (2021) Link between the start of pandemic SARS-CoV/2 (COVID19) and the Human Seafood Wholesale Market in Wuhan (Hubei: China): a first review of bibliographical pre-pandemic evidences.

3. Chukwuma Sr C (2021) Ecosystem cadastre of plant-soil interactions with nonferrous metals. IJCRCPS 8(6): 17-26.

4. Baker RE, Mahmud AS, Miller IF, Rajeev M, Rasambainarivo F, et al. (2021) Infectious disease in era of global change. Nat Rev Microbiol

5. Evans TS, Shi Z, Boots M, Liu W, Olival KJ, et al. (2020) Synergistic ChinaUS ecological research is essential for global emerging infectious disease preparedness. EcoHealth 17: 160-173.

6. Chukwuma Sr C (2021) Accessing vaccines with compliance to stem the emergence and dissemination of the SARS-CoV-2 or COVID-19 variants.

7. Chukwuma Sr C (2021) Challenges and opportunities in accessing veritable vaccines and curbing the dissemination and excesses of the SARS-CoV-2 or COVID-19 variants. Middle East Publishers Scholars International Journal of Biochemistry 4(9): 108-111.

8. Sabin NS, Calliope AS, Simpson SV (2020) Implications of human activities for (re)emerging infectious diseases, including COVID-19. J Physiol Anthropol 39: 29.

9. Chukwuma Sr C (1996) Acquired immunodeficiency syndrome in sub-Saharan Africa: Epidemiological, sociological and preventive perspectives. Scandinavian Journal of Development Alternatives 15(2): 74-89.

10. Greenwood M What is gain-of-function research?

11. Cele S, Gazy I, Jackson L (2021) Escape of SARS-CoV-2 501Y.V2 variants from neutralization by convalescent plasma. Nature

12. (2021) The Economist. Will the COVID-19 pandemic make this year's flu season worse?

13. USRTK.

14. Chukwuma Sr C (2020) Information and communication needs in the administration of ibuprofen in the treatment of symptoms in COVID-19 patients. JMR 6(4): 128-130.

15. Wan Y, Shang J, Graham R, Baric RS, Li F (2020) Receptor recognition by the novel coronavirus from Wuhan: an analysis based on decade-long structural studies of SARS coronavirus. J Virol 94(7).

16. Fang L, Karakiulakis, Roth M (2020) Are patients with hypertension and diabetes at increased risk for COVID-19 infection? Lancet Respir Med 8.

17. Day M (2020) COVID-19: ibuprofen should not be used for managing symptoms, say doctors and scientists. BMJ 368: m1086. 
18. Excler JL, Seville M, Berkley S, Kim JH (2021) Vaccine development for emerging infectious diseases. Nat Med 27: 591-600.

19. Chukwuma Sr C The impacts of mining operations in Nigeria with particular reference to the Enyigba-Abakaliki area. Int J Env Edu \& Inf 12(4): 321-36.

20. Chukwuma Sr C (2014) Information-base and determinants of medical specialization and primary care: A viewpoint. JBAH 4(8).

21. Chukwuma Sr C, Bjurulf P (1990) A case study of the information-base needed for effective evaluation of primary health care programmesin Anambra State, Nigeria. Int J Health Sc 1(2): 137-144

22. Chukwuma Sr C (2021) Ecological analysis of the COVID-19 pandemic for restoration and sustainability. Research and Advances in Pharmacy \& Life Sciences 3(1).

23. Chukwuma Sr C (1996) Defining environmental management and planning policy in Nigeria. Int J Agric Med \& Rural Health 20(1): 13-18.

24. Sawanpanyalert P (2006) Roles of multi-country networking in prevention and control of emerging and re-emerging infections. Trop Med \& Health 34(4): 149-152.

25. Schwartz DA, Herman C (1996) Editorial Response: The importance of the autopsy in emerging and reemerging infectious diseases. Clin Infect Dis 23(2): 248-254.

26. Fidler DP (1996) Germs, governance, and global public health in the wake of SARS. J Clin Invest 1996113: 799-804.

27.https://www.researchgate.net/publication/304635699_The Geopolitics_of_Global_Public_Health_Surveillance_in_the_Twenty-First_ Century.

28. Chukwuma Sr C (2020) Hunger in the COVID-19 pandemic. Int J Adv Res Rev IJARR 5(11): 20-23.

29. Raheem D, Chukwuma Sr C (2001) Foods from cassava and their relevance to Nigeria, and other African countries. Agriculture \& Human Values 18(4): 383-90.

30. Chukwuma Sr C (2019) Restoration and sustainability of petroleumcontaminated sites in the human environment. Journal of Clinical Trials and Regulations 1(1): 57-70.

31. Dodds K, Cole J (2021) Unhealthy geopolitics: Can the response to COVID-19 reform climate change policy? Bull. World Health Organ 99(2): 148-154.

32. Tabor E, Gerety RJ, Cairns J, Bayley AC (1990) Did HIV and HTLV originate in Africa? (Letter). JAMA 264(6): 691-692.

33. Ardoo A (1990) The limits and prospects of AIDS legislation in anglophone Africa. Int-Conf-AIDS 5: 941.

34. (2021) WHO. Africa unlikely to end AIDS as public health threat by 2030.

35. Chukwuma Sr C (1996) Cryptosporidium: Still a public health problem A review. Niger Med J 30(1): 6-10.

\section{ISSN: 2574-1241}

DOI: 10.26717/BJSTR.2022.40.006500

Chrysanthus Chukwuma Sr. Biomed J Sci \& Tech Res

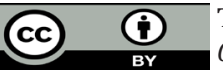

This work is licensed under Creative

Commons Attribution 4.0 License

Submission Link: https://biomedres.us/submit-manuscript.php
36. Chukwuma Sr C (2019) Cryptosporidium: public health problems and environmental indicators. Austin Medical Sciences 4(2): 1036.

37. Chukwuma Sr C (2021) The Clinicopathological correlates of cystoisosporiasis in immunocompetent, immunocompromised and HIV-infected/AIDS patients but neglected in SARS-CoV-2/COVID-19 patients? Int J Clin Microbiol Biochem Technol 4: 001-004.

38. Chukwuma Sr C (1996) Microsporidium in AIDS patients - A perspective. East African Medical Journal 73(1): 74-78.

39. Sen M, Honavar SG, Sharma N, Sachdev MS (2021) COVID-19 eye. A review of ophthalmic manifestations of COVID-19. Ind J Ophthalmol 69(3): 488-509.

40. Chukwuma Sr C (2021) Explicating the presentations of Acanthamoeba keratitis with special concern in the COVID-19 pandemic ambient. IJCMBT 4: 010-015.

41. Jones L, Walsh K, Willcox M, Nichol J (2020) The COVID-19 pandemic: important considerations for contact lens practitioners. Contact Lens Anterior Eye 43(3): 196-203.

42. Chukwuma Sr C (1996) Comments on the environmental health implications of Acanthamoeba. Int J Env Hith Res 6(1): 49-54.

43. Chukwuma Sr C (1996) Application of global concepts to the Nigerian food crisis. Economic Planning in Free Societies. Journal of Agriculture \& Related Studies 1995 31(5): 3-9.

44. (2015) National Academies Press (US). Gain-of-Function Research Background and Alternatives.

45. (2021) News Feature. The shifting sands of "gain-of-function" research Nature.

46. Cao C (2021) China's evolving biosafety/biosecurity legislations. J Law Biosci 8(1).

47. Selgelid MJ (2016) Gain-of-Function Research: Ethical analysis. Sci Eng Ethics 22(4): 923-964.

48. Chukwuma Sr C (1996) Undergirding environmental legislation and citizen participation in developing countries. Int J Env Edu \& Inf 15(3): 319-30.

49. Chukwuma Sr C (1996) Perspectives for a sustainable society. Environ Manage \& Health 7(5): 6-20.

50. Chenneville T, Gabbidon K, Hanson P, Holyfield C (2020) The impact of COVID-19 on HIV treatment and research: A call to action. Int J Environ Res Public Health 17(12): 45-48.

51. Chukwuma Sr C (2021) Accessing vaccines with compliance to stem the emergence and dissemination of the SARS-CoV-2 or COVID-19 variants. In: Chukwuma Sr C (Edt.)., MedDocs Publishers LLC, USA 3(2): 12-15.

52. Morens DM, Fauci AS (2020) Emerging pandemic diseases: How we got to COVID-19. Cell 182(5): 1077-1092.

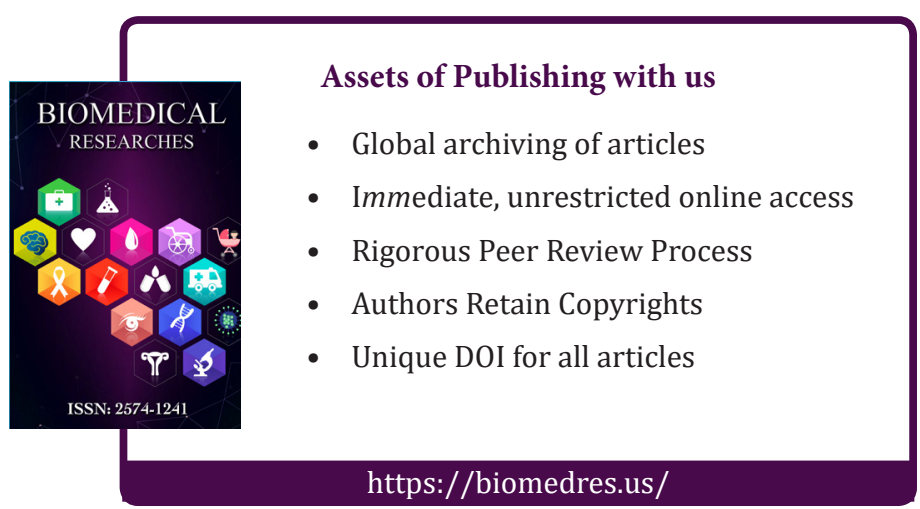

